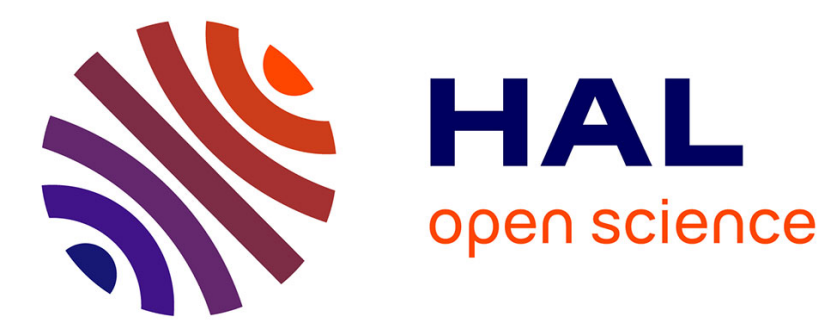

\title{
Signal Quality Monitoring for New GNSS Signals
}

Jean-Baptiste Pagot, Paul Thevenon, Olivier Julien, Francisco

Amarillo-Fernandez, Denis Maillard

\section{To cite this version:}

Jean-Baptiste Pagot, Paul Thevenon, Olivier Julien, Francisco Amarillo-Fernandez, Denis Maillard. Signal Quality Monitoring for New GNSS Signals. Navigation, 2018, Volume 65 (1), pp. 1750-1763. 10.1002/navi.218. hal-01512448

\section{HAL Id: hal-01512448 \\ https://hal-enac.archives-ouvertes.fr/hal-01512448}

Submitted on 17 May 2017

HAL is a multi-disciplinary open access archive for the deposit and dissemination of scientific research documents, whether they are published or not. The documents may come from teaching and research institutions in France or abroad, or from public or private research centers.
L'archive ouverte pluridisciplinaire HAL, est destinée au dépôt et à la diffusion de documents scientifiques de niveau recherche, publiés ou non, émanant des établissements d'enseignement et de recherche français ou étrangers, des laboratoires publics ou privés. 


\title{
Signal Quality Monitoring for New GNSS Signals
}

\author{
J.B. Pagot, P. Thevenon, O. Julien, ENAC, France \\ Francisco Amarillo Fernandez, ESA, the Netherlands \\ Denis Maillard, CapGemini, France
}

\section{BIOGRAPHIES}

Jean-Baptiste Pagot is a PhD candidate in the Department of Signal Processing and Navigation (SIGNAV) at the ENAC (Ecole Nationale de l'Aviation Civile) in Toulouse, France. He received his master degree as an Electronics and Telecommunication engineer in 2013 from the ENAC. He is currently working on GNSS Signal Distortions.

Dr. Paul Thevenon graduated as electronic engineer from Ecole Centrale de Lille in 2004 and obtained in 2007 a research master at ISAE in space telecommunications. In 2010, he obtained a $\mathrm{PhD}$ degree in the signal processing laboratory of ENAC in Toulouse, France. From 2010 to 2013, he was employed by CNES, to supervise GNSS research activities. Since the July 2013, he is employed by ENAC as Assistant Professor. His current activities are GNSS signal processing, GNSS integrity monitoring and hybridization of GNSS with other sensors.

Dr. Olivier Julien is the head of the Signal Processing and Navigation (SIGNAV) research group of the TELECOM laboratory of ENAC, in Toulouse, France. He received his engineer degree in 2001 in digital communications from ENAC and his $\mathrm{PhD}$ in 2005 from the Department of Geomatics Engineering of the University of Calgary, Canada. His research interests are turned towards the use of satellite-based navigation systems for safe navigation.

Francisco Amarillo-Fernández received his Master's Degree in Telecommunication by the Polytechnic University of Madrid (UPM) Spain in 1997, and his Master's Degree in Surveying Engineering by the UPM in 1992. He has been working for the ESA Navigation Directorate since 2001 and has participated/led in numerous research activities in the GNSS field since 1997.

Denis Maillard received his engineer degree in Electronic and Computer Engineering from Institut National des Sciences Appliquées de Rennes in 2010. Since 2011 he works as a GNSS engineer in the EGNOS team of Capgemini Bayonne. He is currently in charge of the activities linked to GNSS signal processing.

\footnotetext{
ABSTRACT

In the context of GNSS signals and associated augmentation systems modernization, new modulations
}

are envisaged. More precisely Galileo E1C, the pilot component of the E1 Open Service signal (CBOC(6,1,1/11) modulation), Galileo E5a and GPS L5 (BPSK(10) modulation) are signals that will be used by civil aviation receivers for pseudorange computation. To meet stringent requirements defined for civil aviation GNSS receivers, the characterization of distortions which could affect a GNSS signal in a hazardous way is required. In particular, expected signal distortions generated at payload level are described by Threat Models (TM). Distortions incorporated in the TM are also called Evil WaveForm (EWF).

These TMs, and their associated parameter ranges, referred to as Threat Space (TS), are powerful and necessary tools to design and test the performance of Signal Quality Monitor (SQM). The SQM is a mean to detect the presence of dangerous signal distortions and is necessary to protect users with high requirements in terms of integrity, accuracy, availability, and continuity (for example civil aviation users). Nowadays, this monitoring task is performed by GBAS and SBAS reference stations for GPS L1 C/A to warn the user in a timely manner. In this paper, SQMs for Galileo E1C and Galileo E5a will be designed and compared by mean of an innovative representation inspired from [1]. From this representation, SQM performance is assessed based on the highest differential tracking error entailed by a signal distortion included in the TM and not detected by the SQM within allocated $P_{f f d}$ and $P_{m d}$. It is noteworthy that performance of SQM is dependent on several parameters and in particular on the $C / N_{0}$ at which the reference station is operating. One of the advantage of the proposed representation is that performances of the SQM can be assessed for different equivalent $C / N_{0}$ from one figure. Using this representation, different SQMs are compared and an optimized SQM is proposed to monitor signal distortions on Galileo E5a and Galileo E1C signals.

\section{INTRODUCTION}

The type of distortion generated by the satellite payload and that could entail large errors on a differential GNSS user without being detected are called Evil WaveForms (EWF) and are a burning issue for GNSS users with strict requirements. In order to represent signal distortions that could be generated by the payload, a proposition of Threat Models (TM) was made in 2001 for GPS L1 C/A [1]. The aim of this TM was to define the type of signal distortion 
that could be created by the GPS satellite payload and that could create a hazard for a civil aviation user. Nowadays, the proposition made in 2001 has been adopted by ICAO with the definition of three threat models for GPS L1 C/A signal [2]:

- TM-A which is associated with a failure in the navigation data unit (NDU), the digital partition of a satellite payload. It consists of the normal C/A code signal except that all the positive chips have a falling edge that leads or lags relative to the correct end-time for that chip $(\Delta)$.

- TM-B which introduces amplitude modulation and models degradations in the analog section of a satellite. More specifically, it consists of the output from a second order system when the nominal C/A code baseband signal is the input. Two parameters are defined for this threat model: the damping factor $\sigma$ and the ringing frequency $f_{d}$.

- $\quad$ TM-C which is a combination of the two first failures.

The advent of new GNSS signals requests new research in the SQM field. Indeed, new signals use different modulations. Consequently TMs have to be redefined and SQM efficiency regarding these new TMs must be assessed. A proposal for Galileo E1C and Galileo E5a signals TM is given in a previous publication [3]. These TMs will be the starting points for the work performed in this publication.

It is noticeable that performances of SQM are dependent upon the TM but they are also dependent upon the receiver configurations to protect. In this document, different user's configurations (correlator spacing, pre-correlation bandwidth and RF filter type) based on expected civil aviation requirements will be taken into account to cover the largest number of possible cases.

The paper content has the following structure:

- The first section will detail the context of this work. Definition of tested TMs and receiver configurations of interest will be given.

- The second section will introduce different metrics based on simple ratio, difference ratio and sum ratio. The methodology to assess SQM performances as a function of metric standard deviation will be described. These performances will be defined in an ideal theoretical way, considering an ideal Gaussian distribution for the noise on correlator outputs [4] and on metrics. A reference SQM based on simple metrics (only correlator ratios) but a high number of available correlator outputs (51 outputs) spaced by 0.01 chip will be first tested on GPS L1 C/A signal. Due to advances in receiver technology, this SQM design is nowadays conceivable. GPS L1 C/A signal will be taken as an example in order to illustrate the concept of an innovative representation to assess performances of SQM. As a conclusion of this part, a method to adapt theoretical results to operating reference station conditions is suggested.
- The third section will compare different performances of SQMs regarding Galileo E1C and Galileo E5a signal deformations. A compromise will be found between SQM complexity and its performances. The sensitivity of the reference SQM will be tested for 3 key parameters: the area covered by the correlators, the spacing between correlators, and the type of metrics (ratio, difference or sum of available correlator outputs). At the end, an optimized SQM (in terms of ratio between performance and complexity) will be proposed for both signals. Optimized SQMs are not optimal and better SQMs could be found using more complex metrics than presented in this paper, as for example the alpha metric used nowadays in WAAS reference stations [5]. Nevertheless proposed optimized SQMs are simple to implement and show sufficient performances regarding the context of this study.

- The last section will conclude on the relevance of the new comparison tool (inspired from [7]) and on the performances provided by proposed SQMs.

The proposition and assessment of SQM techniques adapted to the new Galileo signals is the main objective of this paper. Results obtained for Galileo E5a are also valid for GPS L5, both BPSK(10) modulated.

\section{CONTEXT OF THE STUDY}

As introduced, design and performance of SQM are dependent upon:

- User's configurations to protect and reference station configurations. Receiver parameters of interest at user and reference levels are: the tracking technique (including the local replica modulation), the tracking pair correlator spacing and the RF front-end (technology, bandwidth and maximum group delay variation).

- The TM, or in other words, the distortions that have to be monitored.

As a targeted requirement in this document the maximum tolerable differential error (denoted as MERR in the literature) induced by an undetected distortion of the TM is fixed to 3.5 meters which is a typical value used by civil aviation. Nevertheless from results presented in this study, it is possible to assess SQM performances independently from MERR value. SQM performances are considered as acceptable if the maximum undetected differential error (MUDE) respecting the ICAO requirements for that SQM is below the MERR.

\section{Receiver configurations of interest}

The reference station configuration is fixed: its RF filter is considered as a 6-order Butterworth with a $24 \mathrm{MHz}$ bandwidth (double sides) and its discriminator is an early minus late with a 0.1 chip spacing for Galileo E1C and GPS L1 C/A signals and 1 chip spacing for Galileo E5a signal. Local replicas at reference level are modulated differently depending on the processed signal: BOC(1.1) for Galileo 
E1C, BPSK(1) for GPS L1 C/A and BPSK(10) for Galileo E5a signal.

More configurations are tested at user level as shown in Table 1. These configurations represent receiver architectures expected for civil aviation users [4].

Different types of filters are used, to account for the wide variety of filters encountered across multiple receiver manufacturers. All these filters satisfy ICAO requirements:

- Filter1: 6-order Butterworth (the differential group delay is equal to $39 \mathrm{nsec}$ ).

- $\quad$ Filter2: resonator filter type with a group delay equal to zero.

- Filter3: resonator filter type with a concave group delay and a 150 nsec differential group delay.

- Filter4: 6-order Butterworth for the amplitude and the smallest order Butterworth filter leading to a differential group delay higher than $150 \mathrm{nsec}$ for the phase.

Table 1. Tested user's configurations.

\begin{tabular}{|c|c|c|}
\cline { 2 - 3 } \multicolumn{1}{c|}{} & $\begin{array}{c}\text { Galileo E1C } \\
\text { (CBOC(6,1,1/11)) } \\
\text { and GPS L1 C/A } \\
\text { signal (BPSK(1)) }\end{array}$ & $\begin{array}{c}\text { Galileo E5a } \\
\text { signal } \\
\text { (BPSK(10)) }\end{array}$ \\
\hline $\begin{array}{c}\text { Tracking } \\
\text { technique }\end{array}$ & $\begin{array}{c}\text { E-L (BOC(1.1) and } \\
\text { BPSK(1) local replica) }\end{array}$ & $\begin{array}{c}\text { E-L (BPSK(10) } \\
\text { local replica) }\end{array}$ \\
\hline $\begin{array}{c}\text { Correlator } \\
\text { spacing }\end{array}$ & $0.08,0.1$ and 0.12 chip & $\begin{array}{c}0.8,1 \text { and } 1.2 \\
\text { chip }\end{array}$ \\
\hline $\begin{array}{c}\text { Pre- } \\
\text { correlation } \\
\text { bandwidth } \\
\text { (double sided) }\end{array}$ & $12,14,16,18,20,22,24 \mathrm{MHz}$ \\
\hline $\begin{array}{c}\text { Equivalent RF } \\
\text { filter }\end{array}$ & \multicolumn{2}{|c|}{4 filters are tested } \\
\hline
\end{tabular}

\section{Distortions of interest}

As introduced, for Galileo E5a and Galileo E1C, performance of SQM will be evaluated from TMs proposed in [3]. Regarding GPS L1 C/A TM, the current ICAO TM is kept and is recalled in [2].

Definition of the TMs and associated Threat Space (TS) for the three signals are summarized in Table 2 where:

- $\quad f_{d}$ is the frequency of the ringing phenomenon.

- $\quad \sigma$ is the damping factor of the ringing phenomenon.

- $\quad \Delta$ is the lead/lag at every falling transitions after modulation.

- $\Delta_{11}$ is the lead/lag at every $\operatorname{BOC}(1,1)$ falling transitions at signal square wave generator.

- $\Delta_{61}$ is the lead/lag at every $\operatorname{BOC}(6,1)$ falling transitions at signal square wave generator.

Table 3. Studied TS for GPS LI C/A signal.

\begin{tabular}{|c|c|c|c|}
\cline { 2 - 4 } \multicolumn{1}{c|}{} & $\Delta$ & $\sigma$ & $f_{d}$ \\
chip & MNepers $/ s$ & $M H z$ \\
\hline TM A & {$[-0.12: 0.1: 0.12]$} & - & - \\
\hline TM B & - & {$[0.8: 1: 8.8]$} & {$[4: 1: 17]$} \\
\hline TM C & {$[-0.12: 0.1: 0.12]$} & {$[0.8: 1: 8.8]$} & {$[7.3: 0.57: 13]$} \\
\hline
\end{tabular}

\section{THEORETICAL SQM CONCEPT}

SQM consists of a test (noted Test) to evaluate if the signal is affected by a distortion or not.

SQM methodology has already been described as for example in [4] or [6].

This test compares to a threshold the difference between a current metric value and the metric value in the nominal case. In this document, several metrics are introduced to build the test and metrics are estimated from outputs of the correlation function.

Table 2. Studied TS for new modulations.

\begin{tabular}{|c|c|c|c|c|c|c|c|c|c|c|c|}
\hline & \multicolumn{9}{|c|}{ TM-C } & & \\
\hline & \multicolumn{8}{|c|}{ TM-B } & \multicolumn{3}{|c|}{ TM-A } \\
\hline & \multicolumn{4}{|c|}{ Area 1} & \multicolumn{4}{|c|}{ Area 2} & \multirow{2}{*}{$\begin{array}{c}\text { TM- } \\
\text { A1 } \\
\Delta_{\max } \\
= \\
- \\
\Delta_{\min } \\
\text { chip }\end{array}$} & \multicolumn{2}{|c|}{ TM-A2 } \\
\hline & $\begin{array}{c}f_{d \_ \text {min }} \\
M H z\end{array}$ & $\begin{array}{c}f_{d \_ \text {max }} \\
M H z\end{array}$ & $\begin{array}{l}\quad \sigma_{\min } \\
\text { Mnepers } \\
/ s\end{array}$ & $\begin{array}{l}\quad \sigma_{\max } \\
\text { Mnepers } \\
/ s\end{array}$ & $\begin{array}{c}f_{d \_m i n} \\
M H z\end{array}$ & $\begin{array}{c}f_{d \_ \text {max }} \\
M H z\end{array}$ & $\begin{array}{l}\left(\frac{\sigma}{\left(f_{d}\right)^{2}}\right)_{\min } \\
\text { nepers } / \mathrm{s} \\
/ \mathrm{Hz} / \mathrm{MHz}\end{array}$ & $\begin{array}{l}\left(\frac{\sigma}{\left(f_{d}\right)^{2}}\right)_{\max } \\
\text { nepers } / \mathrm{s} \\
/ \mathrm{Hz} / \mathrm{MHz}\end{array}$ & & $\begin{array}{l}\Delta_{11 \max } \\
= \\
- \\
\Delta_{11 \min } \\
\text { chip }\end{array}$ & $\begin{array}{c}\Delta_{61 \max } \\
= \\
- \\
\Delta_{61 \min } \\
\text { chip } \\
\end{array}$ \\
\hline $\begin{array}{c}\text { Galileo } \\
\text { E1C } \\
\end{array}$ & 1 & 19 & 0 & 26 & 3 & 19 & 0.07 & 5 & 0.12 & 0.1 & 0.08 \\
\hline $\begin{array}{c}\text { Galileo } \\
\text { E5a } \\
\end{array}$ & 3 & 19 & 0 & 24 & 4 & 19 & 0.06 & 3.5 & 1.2 & / & / \\
\hline Resolution & & 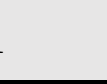 & & & & L & $\begin{array}{r}0.05(\mathrm{Ga} \\
0.075(\mathrm{G}\end{array}$ & $\begin{array}{l}\text { leo E1C) } \\
\text { ileo E5a) }\end{array}$ & 0.01 & 0.01 & 0.01 \\
\hline
\end{tabular}


Mathematically, the test on one metric (noted Test $t_{\text {metric }}$ ) is equivalent to compare the following expression to a given threshold:

$$
\text { Test }_{\text {metric }}=\frac{\text { metric }_{\text {dist }}^{i}-\text { metric }_{\text {nom }}}{\text { threshold }}
$$

Where

- $\quad$ metric $_{\text {dist }}^{i}$ is the current value of the metric which can be affected by a distortion. The index $i$ shows that this value is estimated based on one ranging signal sent by satellite $i$.

- metric $_{\text {nom }}$ is the nominal value of the metric. In theory the nominal value can consist in the median of that metric across all satellites in view in nominal conditions [7]. In practice, because of the difference of correlation function shape in nominal conditions depending on the PRN, metric nom $_{\text {has }}$ to be estimated from the average value of that metric for a given PRN.

\section{Performance threshold estimation}

In order to know if faulty cases are detected with adequate $P_{f f d}$ and $P_{m d}$, a Neyman Pearson hypothesis test is performed. The MDE or MDR (Minimum Detectable Error/Ratio) are performance thresholds that fulfills the ICAO requirements for a test based on only one metric $\left(\right.$ Test $=$ Test $\left._{\text {metric }}\right)$. The definition of the MDE is given based on one metric is given in [2] as:

where

$$
M D E_{\text {metric }}=\left(K_{m d}+K_{f f d}\right) \sigma_{m e t r i c}
$$

- $\quad K_{f f d}=5.26$ is a typical fault-free detection multiplier representing a false detection probability of $1.5 \times$ $10^{-7}$ per test;

- $\quad K_{m d}=3.09$ is a typical missed detection multiplier representing a missed detection probability of $10^{-3}$ per test;

- $\sigma_{\text {metric }}$ is the standard deviation of measured values of the test metric;

For the above expression to hold, it is assumed that the noise affecting metrics is white and Gaussian. The Gaussian behaviour of the noise affecting correlator outputs was verified in [4]. Details are given about the Gaussian assumption of metrics in the following.

If several metrics are used, as it is envisaged in this paper, $P_{f f d \_m e t r i c}$ and $P_{m d \_m e t r i c}$ have to be computed for each individual metric. Indeed, the proposed SQM test is based on several partially dependent sub-tests.

Let us call $P_{X_{-} \text {metric }}(X=f f d$ or $m d$ ) the probability associated to one test based on one metric and let us assume that the same budget is allocated to each sub-test. Considering that the total test is based on $N_{\text {test }}$ sub-tests and that an alarm is triggered if at least one metric exceeds its threshold, two extreme cases are imaginable:

- Metrics are totally independent. In this condition probabilities of one sub-test $\left(P_{X_{-} \text {metric }}\right)$ are related to the total test probabilities $\left(P_{X}\right)$ by:

$$
\begin{gathered}
P_{f f d}=\sum_{1}^{N_{\text {test }}}\left(C_{N_{\text {test }}}^{k} P_{f f d_{\text {metric }}}^{k}\right. \\
\left.\left(1-P_{f f d \_m e t r i c}\right)^{N_{\text {test }}-k}\right) \\
P_{m d}=P_{m d_{-} \text {metric }} N_{\text {test }}
\end{gathered}
$$

- Metrics are totally dependent, in this case:

$$
\begin{aligned}
P_{f f d} & =P_{f f d \_m e t r i c} \\
P_{m d} & =P_{m d_{-} \text {metric }}
\end{aligned}
$$

In real conditions, $P_{X_{-} \text {metric }}$ are between the two extrem cases defined by Equation (2), (3), (4) and (5) . With a more precise knowledge about the relation dependency between each metric, exact $P_{X_{-} \text {metric }}$ and consequenlty exact $\left(K_{m d}+K_{f f d}\right)$ could be estimated. $\left(K_{m d}+K_{f f d}\right)$ is assessed in this document in a conservative way which is obtained when metrics are considered as totally dependent. It entails that even if several metrics are used to define a testthe MDE fulfilling the ICAO requirements in terms of $P_{m d}$ and $P_{f f d}$ can be modelled in a conservative way, on each metric, as:

$$
M D E_{\text {metric }}=8.35 \times \sigma_{\text {metric }}
$$

The three types of metric used in this document are elementary and are presented in Equations (7), (8) and (9). These metrics are looked at for two main reasons:

- the simple ratio and the difference ratio metrics are currently used in SQM implemented in EGNOS.

- the value of $\sigma_{\text {metric }}$ for these three metrics can be derived theoretically in a simple way.

Simple ratio metric which is the easiest metric to implement and permits to detect all kind of correlation function deformations.

$$
\operatorname{metric}_{x}=\frac{I_{x}}{P}
$$

Difference ratio metric which permits to detect distortions that affect the correlation function in an asymmetric way (asymmetric from the prompt) more efficiently than the simple ratio metric.

$$
\operatorname{metric}_{x-x}=\frac{I_{-x}-I_{x}}{P}
$$

And sum ratio metric which permits to detect distortions that affect the correlation function in a symmetric way (symmetric from the prompt) more efficiently than the simple ratio metric.

$$
\operatorname{metric}_{x+x}=\frac{I_{-x}+I_{x}}{P}
$$

where

- $\quad I_{x}$ is the in phase correlator output value at a distance $x$ (in chip unit) from the prompt.

- $\quad P=I_{0}$ is the value of the prompt correlator output. Usually $P=I_{0}(x=0)$. 
An important remark is that in WAAS reference stations, a virtual prompt is used [6]. In this case, instead of $P=I_{0}$, $P=\frac{I_{z}+I_{-Z}}{2}$ with $z=0.025 T_{c}$ is adopted. In this document it is decided to establish results based on the virtual prompt $P=\frac{I_{z}+I_{-z}}{2}$ with $z=0.025 T_{c}$ for GPS L1 C/A and Galileo E1C and with $z=0.25 T_{c}$ for Galileo E5a. This choice has consequences on SQM performances even if repercussions are minor.

Metrics value can then be compared to its nominal value and finally divided by the MDE associated to that metric. Let us define the performance test Test $_{\text {metric_MDE }}$ as:

$$
\begin{aligned}
& \text { Test }_{\text {metric_MDE }} \\
& =\frac{\text { metric }_{\text {dist }}^{i}-\text { metric }_{\text {nom }}}{M D E_{\text {metric }}}
\end{aligned}
$$

If Test $_{\text {metric_MDE }}>1$, this means that a given distortion is detected with the appropriate ICAO $P_{f f d}$ and $P_{m d}$ since it entails a bias larger than the $M D E_{\text {metric }}$. The estimation of $M D E_{\text {metric }}$ is consequently of a primary importance to establish SQM performances.

As discussed previously, $M D E_{\text {metric }}$ is a function of $\sigma_{\text {metric }}$ making the assumption that the noise distribution on metrics is Gaussian.

$\sigma_{\text {metric }}$ can be estimated theoretically for the three introduced metrics. Mathematical $\sigma_{\text {metric }}$ expressions are given in the Appendix and are valid when the noise distribution on metrics (for example $\frac{I_{x}}{I_{y}}$ ) is Gaussian. This condition is fulfilled when:

- $\quad I_{x}$ and $I_{y}$ are treated as Gaussian variables $N\left(\mu_{x}, \sigma_{x}^{2}\right)$ and $N\left(\mu_{y}, \sigma_{y}^{2}\right)$. This properties is acquired and is used in many publications as for instance in [4], [8] or [9].

- $\quad \frac{\mu_{x}}{\sigma_{x}}$ and $\frac{\mu_{y}}{\sigma_{y}}$ tends to infinity. In this case, the ratio of the two Gaussian distributions tends to a Gaussian distribution instead of a more complex distribution [7]. It can be considered that these conditions are verified at reference station level [4] operating with a $1 \mathrm{sec}$ correlation duration.

The theoretical $\sigma_{\text {metric }}$ matches with the $\sigma_{\text {metric }}$ estimated from a Matlab software. This software generates a noisy filtered signal and a local replica. Then the two signals are correlated to obtain a noisy correlation function. Several random draws are generated and metrics are built from noisy correlation functions. Then $\sigma_{\text {metric }}$ can be assessed by simulation. As an example theoretical and simulated $\sigma_{\text {metric }}$ values are compared on Figure 1 . In this particular case, the coherent integration time is equal to $1 \mathrm{sec}$, the $C / N_{0}=30 \mathrm{dBHz}$ and the signal is filtered by the reference filter.

On Figure 1 are presented theoretical (continuous plot) and simulated (dotted plot) metrics standard deviations for metric $_{x-x}$ (in blue), metric $c_{x+x}$ (in purple) and metric $c_{x}$ (in blue for $x$ negative and in purple for $x$ positive). The normalization is based on the virtual prompt. Similar adequacies between theoretical and simulated metrics standard deviations have been obtained for Galileo E1C and E5a signals.
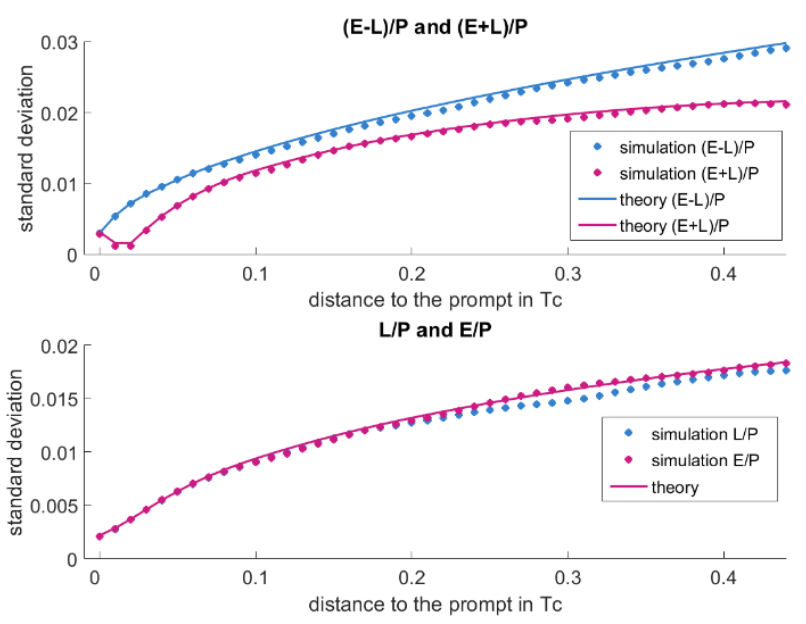

Figure 1. Theoretical and simulated metric standard deviations on a BPSK(1) autocorrelation function.

\section{A representation to assess theoretical performance of SQM, example of GPS L1 C/A signal}

In this document, performance of SQM is assessed based on the highest differential error entailed by an undetected distortion from a given TM considering only the steady state. Knowing the distortion and the value of $M D E_{\text {metric }}$ it is possible to assess Test metric_MDE $_{\text {for each metric and }}$

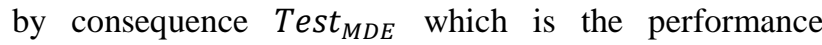
threshold test of a SQM based on several metrics.

Let us denote:

$$
\begin{aligned}
& \text { Test }_{M D E} \\
& =\max _{\text {metric_MDE }}\left[\text { Test }_{\text {metric_MDE }}\right]
\end{aligned}
$$

Then, Test $_{M D E}$ can be estimated as the maximum value among all Test metric_MDE $_{\text {. Comparing Test }}$. TDE $_{\text {to }}$, it is possible to know if a distortion from the TM is theoretically detected with a given $P_{f f d}$ and $P_{m d}$ by a SQM for a given reference station configuration. Moreover, assuming user receiver configurations that have to be protected and the reference station configuration, the highest differential error induced by a given distortion of the TM between different users and the reference can be assessed independently from the SQM. This highest differential error is called the maximum differential error.

Using simulations, Test $_{M D E}$ and maximum differential error values can be estimated for each distortion of the TM. Test $_{M D E}$ is independent from users to protect and depends upon:

- The reference receiver configuration.

- $\quad$ The SQM design implemented on the reference.

- The $C / N_{0}$ of incoming signals which will have a direct impact on $\sigma_{\text {metric }}$ and consequently on $M D E_{\text {metric }}$ and Test $t_{\text {metric_MDE }}$

The reference receiver configuration is fixed with parameter values defined in the previous section. 
A reference SQM is used: it consists of 50 simple ratio metrics. More precisely, 50 metric $_{x}$ are tested for $x=$ -0.25 : 0.01: -0.01 and $x=0.01: 0.01: 0.25$ in chip unit. Normalization of these metrics is based on a virtual prompt which uses correlator outputs at \pm 0.025 chip. Correlator outputs are estimated from a coherent integration time equal to $1 \mathrm{sec}$ and metrics are not smoothed.

Figure 2 shows the maximum differential error induced by distortions from the TM defined by ICAO for GPS L1 C/A signal among the tested user configurations, as a function of the Test $t_{M D E}$ value. The $C / N_{0}$ of the incoming signal is equal to $35 \mathrm{dBHz}$. This representation is comparable to the representation proposed in [1] except that in this document, the value of Test ${ }_{M D E}$ is based on the $P_{m d}$ and $P_{f f d}$ whereas in [1] the value of Test ${ }_{M D E}$ is derived only from the $P_{f f d}$. Each point of the graph corresponds to one distortion of the TM with on the $y$-axis the highest impact on tested users and on the $\mathrm{x}$-axis the value of Test $_{M D E} .1650$ distortions are represented (12 from TM-A, 126 from the TM-B and 1512 from the TM-C). The continuous line corresponds to the higher bound.

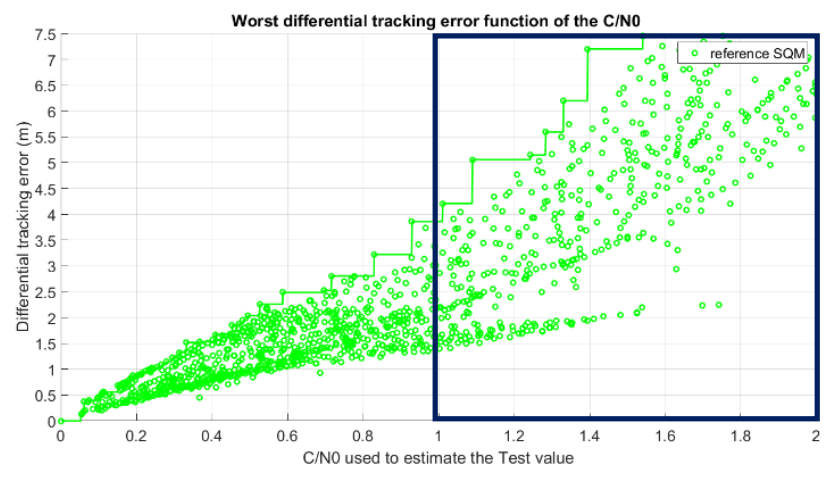

Figure 2. Example of worst differential tracking error function of $\boldsymbol{T e s t}_{\boldsymbol{M D E}}$.

Distortions included in the blue square of Figure 2 are distortions detected by the defined SQM $\left(\right.$ Test $\left._{M D E}>1\right)$ in the described particular case. The Maximum Undetected Differential Error (MUDE) can then been read by taking the largest differential tracking error for Test ${ }_{M D E}<1$. In conditions of Figure 2, the MUDE is equal to $3.9 \mathrm{~m}$.

It is noticeable that MUDE is dependent upon the $C / N_{0}$ which is a drawback because MUDE has to be re-estimated depending on the $C / N_{0}$ at which a reference station is operating. In this document it is proposed to adapt the scale on the $\mathrm{x}$-axis in order to have one representation that permits to assess performances of a given SQM at different $C / N_{0}$.

MUDE is dependent upon the $C / N_{0}$ but a relation exists between $C / N_{0}$ and the value of Test $_{M D E}$. Indeed, $C / N_{0}$ has an impact on $\sigma_{\text {metric }}$ which can be theoretically estimated. Then a relation exists between $\sigma_{\text {metric }}$ and $M D E_{\text {metric }}$, it means between $\sigma_{\text {metric }}$ and Test ${ }_{M D E}$. The relation between $C / N_{0}$ in $\mathrm{dBHz}$ and $\sigma_{\text {metric }}$ is given by:

$$
\sigma_{\text {metric }}=C_{\text {metric }} \sqrt{\frac{1}{T_{\text {int }} \times 10^{\frac{C / N_{0}}{10}}}}
$$

Where $T_{\text {int }}$ is the coherent integration time chosen for the tracking $\left(T_{\text {int }}=1 \mathrm{sec}\right) . C_{\text {metric }}$ is a parameter that does not depend upon the $C / N_{0}$ but depends upon the metric.

By consequence, it is possible to apply a scale change on Figure 2 in order to have the worst differential tracking bias function of Test $_{M D E}=1$ for different $C / N_{0}$. Figure 3 shows same results as on Figure 2 with a simple scale change. The blue square is still representing distortions detected by the SQM considering $C / N_{0}=35 \mathrm{dBHz}$.

One interest of the representation shown in Figure 3 is that MUDE can be assessed for different $C / N_{0}$ from one figure.

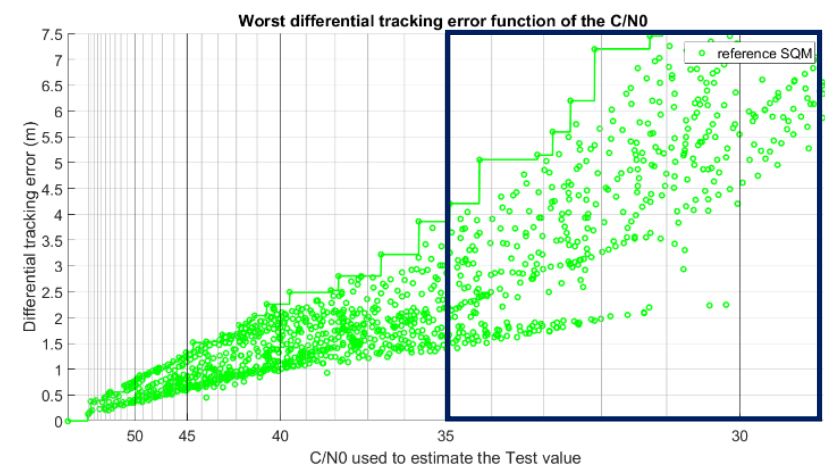

Figure 3. Example of worst differential tracking error function of the equivalent $\boldsymbol{C} / \boldsymbol{N}_{\mathbf{0}}$.

A second interest of the representation is that different performances of different SQMs can be compared. Now, a second SQM is introduced: SQM2b. This SQM was studied around 2000 for example in [5] or [10], and is still used nowadays in EGNOS RIMS-C stations [11]. Originally SQM2b consists of 11 metrics but only 4 metrics are used by RIMS-C stations. The studied SQM is based on the four SQM2b metrics:

$$
\begin{gathered}
\text { metric }_{-0.075}, \text { metric }_{0.075} \\
\text { metric }_{0.075-0.075}, \text { metric }_{0.1-0.1}
\end{gathered}
$$

Figure 4 gives in red results obtained using the SQM2b and in green using the reference SQM based on 50 simple ratio metrics.

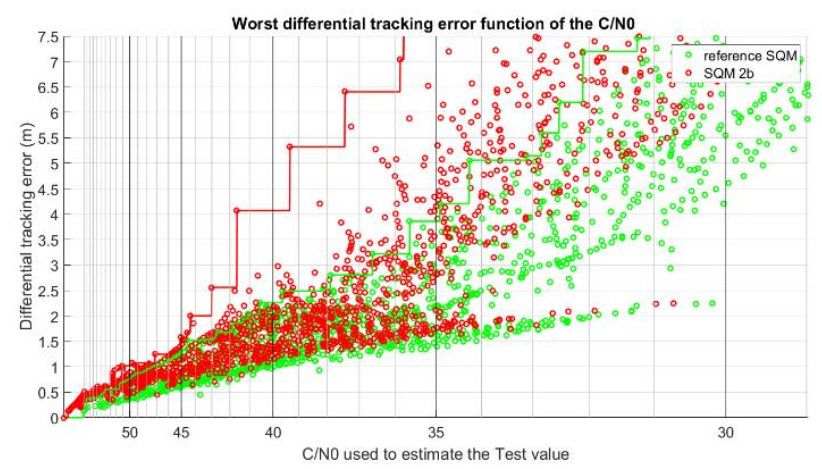

Figure 4. Comparison of two SQMs performance.

From these plots, it is clear that MUDE is higher for the $\mathrm{SQM} 2 \mathrm{~b}$ than for the reference SQM whatever the $C / N_{0}$ is because the red line is above the green line. This result was expected especially because the reference SQM relies on 
50 metrics whereas SQM2b relies on only 4 metrics. The MERR was fixed to 3.5 meters for GPS L1 C/A civil aviation's operations. It can be seen that MUDE is lower than 3.5 meters with the SQM2b only if the $C / N_{0}$ is higher than $42 \mathrm{dBHz}$. This result put forward that with this simulation setup, SQM2b does not reach required performances for signal with a $C / N_{0}$ lower than $42 \mathrm{dBHz}$. This can be interpreted as a reason why a supplementary step in SQM design was proposed in the early 2000s [5]. In order to decrease the metrics standard deviation, it was proposed to smooth metrics values by the mean a low pass digital filter with a time constant equal to or shorter than $100 \mathrm{sec}$ [2]. Such a smoothing was implemented on reference stations as define in [6], [11] or [12]. In theory this smoothing gives the possibility to divide by a factor 10 metric standard deviation. However in practice, especially because of multipath, such improvement is not reachable. In the next section a method is developed to estimate at which equivalent theoretical $C / N_{0}$ a reference station is operating.

\section{Equivalent theoretical $C / N_{0}$ for a reference station in operational conditions}

Results that are presented in the previous section are estimated in ideal conditions:

- The noise distribution on metrics is white and Gaussian.

- The coherent integration time is equal to $1 \mathrm{sec}$.

- No multipath is affecting the incoming signal.

- A 6-order Butterworth (24 MHz double sided) is implemented at the reference level.

To estimate performances of SQM in these ideal conditions, it is necessary to know at which $C / N_{0}$ the worst differential tracking error has to be assessed. It is assumed that an integration time change and the presence of multipath do not have any influence on the Gaussian feature of the noise distribution on metrics: only, the standard deviation of the Gaussian distribution is impacted by the multipath and the integration time. Note that if the noise distribution on metrics is not Gaussian, it is still possible to assess performances of SQM as detailed in the next section.

To estimate the $C / N_{0}$ in reference stations operating condition, $\sigma_{\text {metric }_{x}}$ has to be first estimated for this particular condition. Then an abacus is plotted as shown in Figure 5 and gives an equivalence between $\sigma_{\text {metric }}$ assessed in real conditions and the theoretical $C / N_{0}$ which would permit to obtain that same $\sigma_{\text {metric }_{x}}$ value if only thermal noise was present.

On the abacus on Figure 5, three examples are proposed and represent some real reference stations conditions. It shows $\sigma_{\text {metric }}$ normalized by the virtual prompt $(P=$ $\frac{I_{z}+I_{-Z}}{2}$ with $z=0.025 T_{c}$ ). The two first cases correspond to a data collection performed at Stanford University with a LAAS integrity test-bed on SV 5 with a $5^{\circ}$ elevation angle [5]. Red dots correspond to unsmoothed metrics and green dots to metrics smoothed by a $100 \mathrm{sec}$ moving average. The last case illustrates $\sigma_{\text {metric }}$ obtained from a data collection made by Capgemini with a Novatel GIII receiver. The data collection was 1 hour long and $\sigma_{\text {metric }}$ was estimated from all satellites in view. The worst $\sigma_{\text {metric }}$ among satellites is represented by blue dots. The worst case was observed on SV 62. Its elevation angle was equal to $9^{\circ}$ at the beginning of the data collection and $33^{\circ}$ at the end.

From Figure 5, it can be approximated that the LAAS receiver is working at an equivalent theoretical $C / N_{0}=$ $34 \mathrm{dBHz}$ in the worst case if metrics are unsmoothed whereas the equivalent theoretical $C / N_{0}$ is equal to $38 \mathrm{dBHz}$ with smoothed metrics. With unsmoothed metrics, Capgemini standard deviations correspond in the worst case to a theoretical $C / N_{0}=35 \mathrm{dBHz}$.

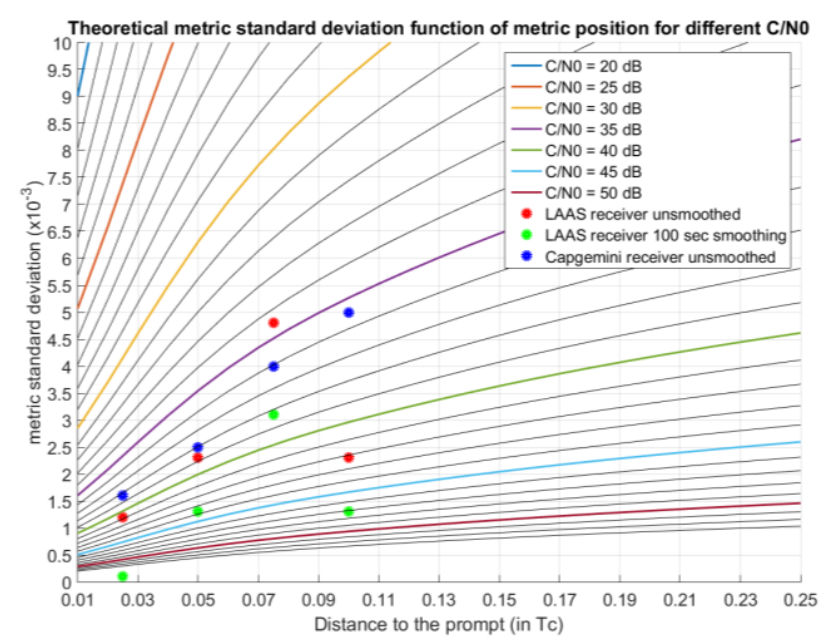

Figure 5. Example of reference station metric standard deviations compared to theoretical values.

One important remark is that the 100 seconds smoothing entails in practice only a $4 d B$ improvement whereas a $10 d B$ improvement is expected in theory. Indeed, as introduced previously, in theory the smoothing could induce a division by a factor 10 of metrics standard deviation. In practice, notably because of multipath, the factor to apply is equal to 1.5. Even if this factor was equal to 2.3 on some signals collected by Capgemini with low $C / N_{0}$, to be conservative, the value of $1.5(4 \mathrm{~dB})$ is considered in the continuation.

Abacuses for Galileo E5a and Galileo E1C signals are provided in Appendix.

\section{Generalization to non-Gaussian noise distribution on metrics}

In order to apply the theoretical SQM concept developed in this paper, the noise distribution on metrics has to be white and Gaussian. In real conditions it can appear that this hypothesis is not true [13]. One of the consequence is that at each reference station, metric performance 
thresholds have to be adjusted to satisfy $P_{m d}$ and $P_{f f d}$. Indeed in this condition, it is not possible to estimate the $M D E_{\text {metric }}$ multiplying $\sigma_{\text {metric }}$ by a factor multiplier derived from normal law. Nevertheless with the knowledge of metrics performance thresholds it is possible to evaluate the equivalent $C / N_{0}$ to apply to a reference station with metrics affected by a not Gaussian noise.

To make this evaluation possible, the abacus on Figure 6 gives an equivalence between $M D E_{\text {metric }}$ assessed in real reference station conditions and the theoretical $C / N_{0}$ which would permit to obtain theoretically the $M D E_{\text {metric }}$ value. The abacus on Figure 6 is equal to the abacus on Figure 5 multiplied by $\left(K_{m d}+K_{f f d}\right)=8.35$.

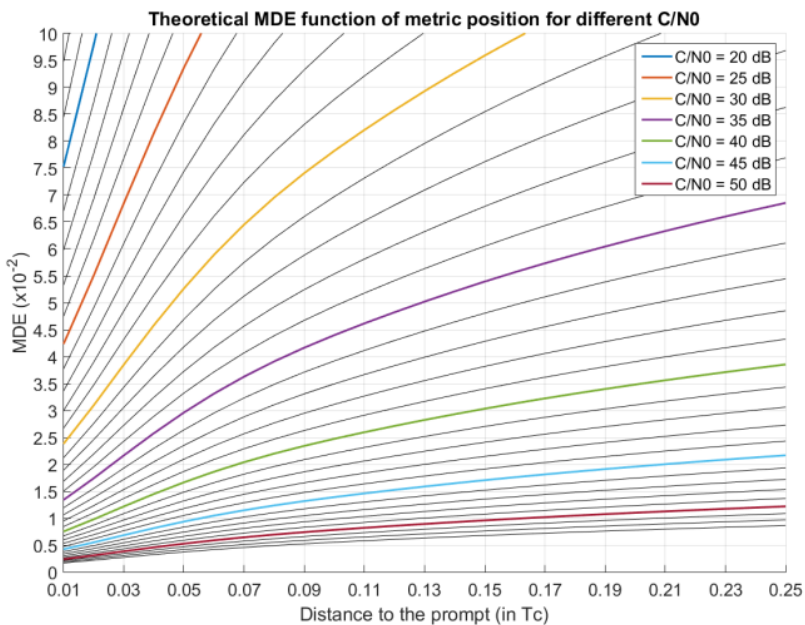

Figure 6. Simple ratio metric performance thresholds for different $C / N_{0}$ and different distance to the prompt.

\section{Conclusion about SQM methodology}

As a conclusion of this section an innovative method was proposed to estimate and compare performances of SQM independently from the equivalent theoretical $C / N_{0}$ at which a reference station operates. Performances are assessed considering that a 6-order Butterworth $(24 \mathrm{MHz}$ double sided) is implemented at the reference level. If this condition is not verified, plots representing worst differential tracking errors function of $C / N_{0}$ are different from plots presented in this publication and have to be assessed for the reference receiver filter of concern.

Considering that no smoothing is applied on metrics, the equivalent theoretical $C / N_{0}$ can be as bad as $34 \mathrm{dBHz}$. In this condition and with a 6-order Butterworth implemented at the reference level, the maximum undetectable differential error (MUDE) is higher than 7.5 meters with SQM2b and is equal to 5.1 meters with the reference SQM (50 simple ratio metrics spread uniformly around the correlation function peak). As an order of magnitude, if a $100 \mathrm{sec}$ moving average window is used to smooth metrics, the equivalent theoretical $C / N_{0}$ can be improved by $4 \mathrm{dBHz}$. In these theoretical conservative circumstances the MUDE is equal to 5.3 meters with the SQM2b and 2.5 meters with the reference SQM.
From these results, it can be deduced that SQM2b is not fulfilling requirements regarding proposed analysis. It could be a reason why SQM2b was replaced by the alpha metric in WAAS reference stations [5]. More reasonably, results provided in this document are conservative. From the theoretical point of view, 4 filters with different bandwidths are taken into account and metrics are considered as totally dependent. With less or different filters to estimate the MUDE and without the assumption of totally dependent metrics, performances of SQM could be improved. From a practical point of view, worst cases are looked at. SQM performances could be increased by the mitigation of the multipath or/and the setting of a mask angle (as example to $15^{\circ}$ (instead of $5^{\circ}$ ) in order to avoid multipath and low $C / N_{0}$ ) and increase the benefit of smoothing.

\section{RESULTS ON NEW SIGNALS}

In this section, SQM theoretical performances are estimated and compared using the method developed in the previous part. Several SQMs are tested in order to find an optimized SQM: reduce the number of sub-tests and obtain suitable performances. A particular care is taken to obtain a MUDE smaller than 3.5 meters for the lowest $C / N_{0}$ as possible. This study is performed on Galileo E1C and Galileo E5a. The first step consists of reducing the area covered by monitored correlator outputs considering only simple ratio metrics to see which part of the correlation function is more affected by distortion. The second step consists of limiting the distance between two monitored outputs to see with which resolution the correlation function has to be monitored. The third step consists of introducing the difference and the sum ratio metrics to see the benefit of using more complex metrics.

In this paper SQM designs for Galileo E1C are based on 51 monitored correlator outputs $I_{x}$ with $x=$ -0.25: 0.01: 0.25 in Galileo E1C chip unit and for Galileo E5a designs are based on 21 correlator outputs $I_{x}$ with $x=$ -1: 0.1: 1 in Galileo E5a chip unit. The limitation to these correlator outputs is justified by three main reasons:

- ICAO TM like distortions are more visible around the prompt of the correlation function. It is not necessary to monitor the correlation function at a too important distance from the prompt.

- Correlator outputs situated at a too important distance from the prompt are more subject to multipath. With the selected correlator output range, the impact of multipath is limited.

- A time delay of $10 \mathrm{nsec}$ between two correlator outputs is nowadays reachable but lower values of time delays are more difficult to achieve.

Three metrics are tested (simple, difference and sum ratio metrics) for all monitored correlator outputs. 


\section{Galileo E1C}

More distortions are tested on new signals because of the larger threat space presented in Table 2. For Galileo E1C, 27314 distortions are generated (12 TM-A1, 80 TM-A2, 494 TM-B on area1, 1600 TM-B on area2, 5928 TM-C on area1 and 19200 TM-C on area2).

Influence of the monitored area size.

On Figure 7 the reference SQM (in green) is compared to two SQMs covering different areas:

- SQM20: it consists of 40 metric $_{x}$ with $x=$ -0.20 : 0.01: -0.01 and $x=0.01: 0.01: 0.20$ in chip unit. (in red)

- SQM13: it consists of 26 metric $_{x}$ with $x=$ -0.13 : $0.01:-0.01$ and $x=0.01: 0.01: 0.13$ in chip unit. (in blue)

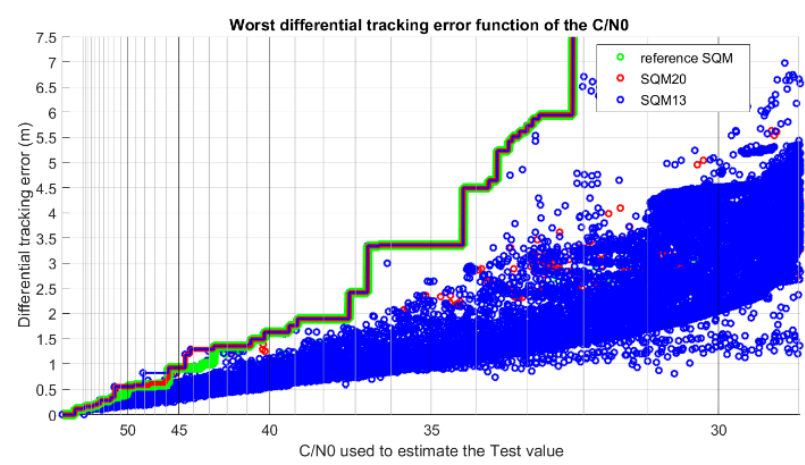

Figure 7. Galileo E1C simple ratio SQMs comparison on different areas.

Results for the reference SQM, the SQM20 and the SQM13 are almost identical and consequently superimposed. It means that benefit of using correlator outputs with a distance to the prompt higher than 0.13 chip is marginal. It is then possible to reduce monitored area to \pm 0.13 chip around the correlation function main peak if simple ratio metrics are considered.

Influence of the distance between two monitored points. Figure 8 shows the influence of the distance between two monitored correlator outputs. Monitors are still uniformly distributed. The comparison is made from three SQMs:

- SQM13 (in green)

- SQM13/02: it consists of 14 metric $_{x}$ with $x=$ -0.13: 0.02: 0.13 in chip unit. (in red)

- SQM13/03: it consists of 10 metric $_{x}$ with $x=$ -0.13 : 0.03: -0.01 and $x=0.01: 0.03: 0.13$ in chip unit. (in blue)

From Figure 8 is can be deduced that it is not necessary to have a too dense monitored correlator outputs distribution. Using a distance between monitors equal to $0.02 T_{c}$ gives approximately same results than using a distance equal to $0.01 T_{c}$. Nevertheless if the distance is equal to $0.03 T_{c}$ SQM performances are degraded. Consequently it seems appropriate to consider that SQM13/02 reaches almost the same performances as the SQM13 and the reference SQM even if it consists of only 14 metrics.

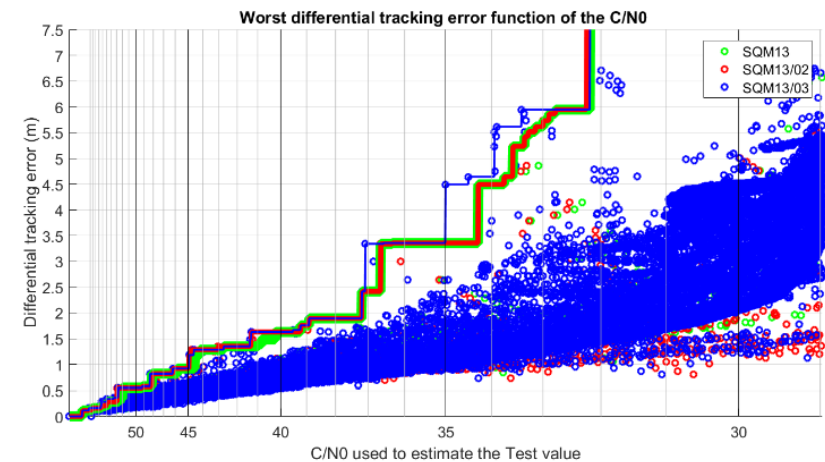

Figure 8. Galileo E1C, comparison of SQMs based on simple ratio metrics for different monitors distributions.

Influence of sum and difference ratio metrics.

Figure 9 illustrates the comparison between three SQMs using more complex metrics:

SQM13/02 (in green)

- SQMsum25: it consists of 25 metric $_{x+x}$ with $x=$ 0.01: 0.01: 0.25 in chip unit. (in red)

- SQMdiff25: it consists of 25 metric $_{x-x}$ with $x=$ 0.01: 0.01: 0.25 in chip unit. (in blue)

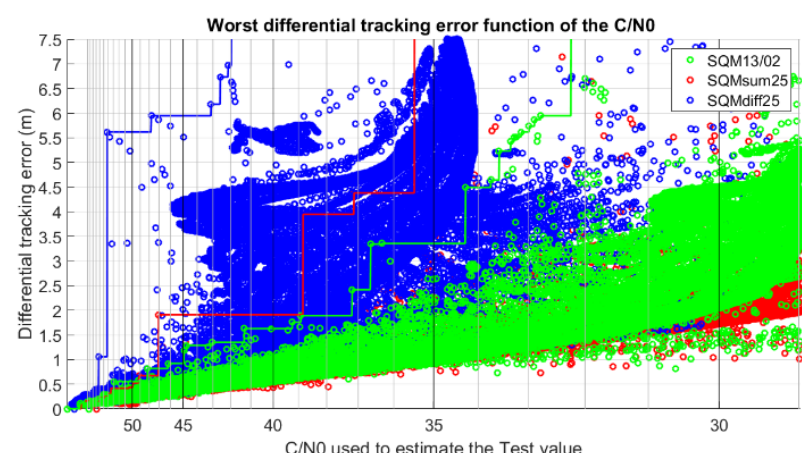

Figure 9. Galileo E1C, comparison of SQMs based on simple, difference and sum ratio.

It can be seen that the SQM based on simple ratio metrics is better than SQM based on more complex metrics. It is noticeable that metrics built on the difference of correlator outputs show bad performance. The problem is that distortions which affect in the same way both sides of the correlation function (symmetry from the prompt), are more difficult to detect with metric m $x-x$ despite the fact that these distortions can lead to high differential error. Example of distortions difficult to detect by any of the 25 metric $_{x-x}$ are illustrated in Figure 10.

In blue is presented a TM-A 2 distortion with $\Delta_{61}=0$ chip , $\Delta_{11}=0.9$ chip inducing a maximum differential error equal to 5.0 meters. In black is a TM-B distortion with $f_{d}=$ $2 \mathrm{MHz}, \quad \sigma=0.07$ nerpers $/ \mathrm{s}$ inducing a maximum differential error equal to 9.5 meters. In red is shown a TMC distortion with $f_{d}=9 \mathrm{MHz}, \sigma=2 \operatorname{nerpers} / \mathrm{s}, \Delta=$ 0.12 chip inducing a maximum differential error equal to 5.6 meters. In green is represented the ideal correlation function. All correlation functions are filtered by the reference filter. 


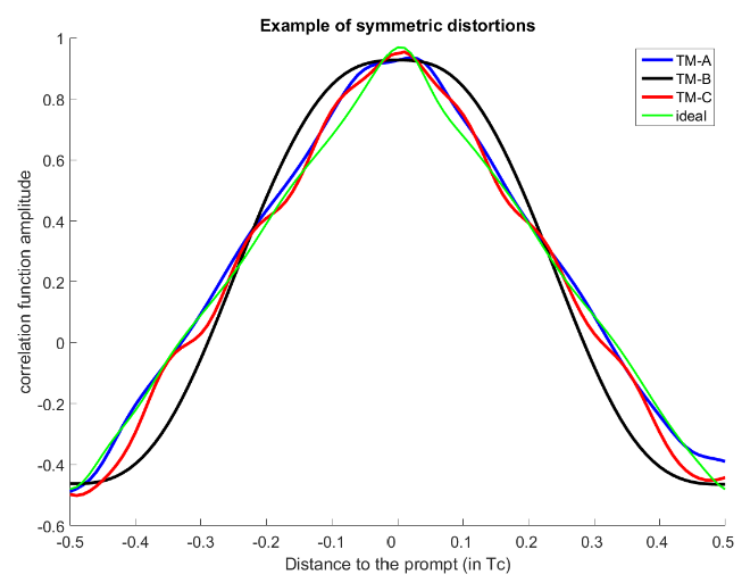

Figure 10. Example of symmetric distortions on Galileo ElC difficult to detect with difference ratio metrics.

Even if some threatening distortions are not detected by more complex metrics, the adoption of these metrics jointly with the SQM13/02 can improve its performance as illustrated on Figure 11. The comparison of SQM13/02 only (in green) with the SQM13/02 + SQMsum25 (in blue) as well as the comparison between SQM13/02 + SQMsum25 (in red) with SQM13/02 + SQMsum25 + SQMdiff25 (in black) show that difference ratio metrics improved slightly performance of the SQM at high $C / N_{0}$ $\left(C / N_{0}>38 \mathrm{dBHz}\right.$ ). As a consequence, it is decided to introduce a difference ratio metric. From Figure 11 the addition of SQMsum 25 grants an evident improvement of SQM efficiency.

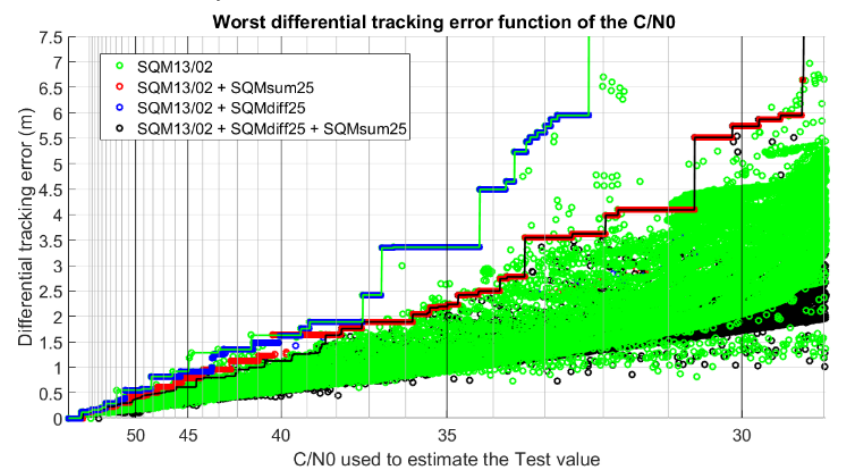

Figure 11. Galileo E1C combination of simple, difference and sum ratio $S Q M$ s comparison.

Finally from Figure 11 and based on results not presented in this paper, it is decided to use SQM13/02 jointly with some sum ratio metrics and one difference ratio metric for the design of the optimized SQM.

\section{Proposed optimized SQM}

As already done in this section for metric $x$, it can be established that it is not necessary to test metric $c_{x+x}$ for all $x=0.01: 0.01: 0.25$. On Figure $12 \mathrm{SQM} 13 / 02$ is used together with:

- SQMsum25 (in green)

- SQMsum13/02: it consists of 7 metric $_{x+x}$ with $x=$ 0.01: 0.02: 0.13 in chip unit. (in red)
- SQMsum13/02 and SQMdiff1: it consists of 7 metric $_{x+x}$ with $x=0.01: 0.02: 0.13$ in chip unit plus 1 metric metric m-x $_{x-x}$ with $x=0.01$. (in blue)

Worst differential tracking errors entailed by undetected distortions regarding the $3 \mathrm{SQMs}$ are practically identical from Figure 12 (the three limits are superimposed). To reduce the number of metrics maintaining satisfying performances, SQM13/02 can be used together with SQMsum13/02 and SQMdiff1.

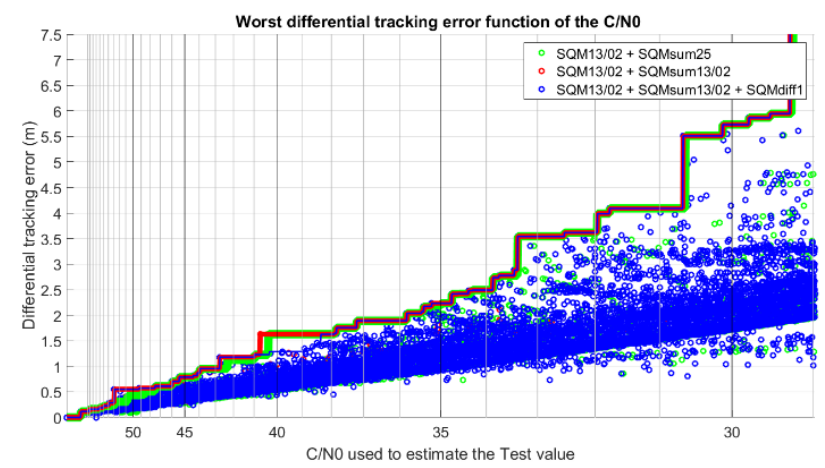

Figure 12. Different simple ratio metrics used with sum ratio metrics. Galileo E1C.

To conclude, an optimized SQM $\left(S Q M_{E 1 C_{\text {optimized }}}\right)$ is proposed to monitor Galileo E1C signal distortions defined previously.

$S Q M_{E 1 C_{\text {optimized }}}$ consists of 14 metric $_{x}$ with $x=$ -0.13: 0.02: 0.13 in chip unit, 7 metric $_{x+x}$ with $x=$ 0.01: 0.02: 0.13 in chip unit and 1 metric metric $_{x-x}$ with $x=0.01$.

This SQM is not optimal but limits the number of sub-tests to realize, almost reaching performances of a SQM based on 50 metric $_{x}$ plus 25 metric $_{x+x}$ plus 25 metric $_{x-x}$. To underline this fact, Figure 13 proposes a performance comparison between $S Q M_{E 1 C_{\text {optimized }}}$ (in red) and the SQM using the 100 metrics (in green).

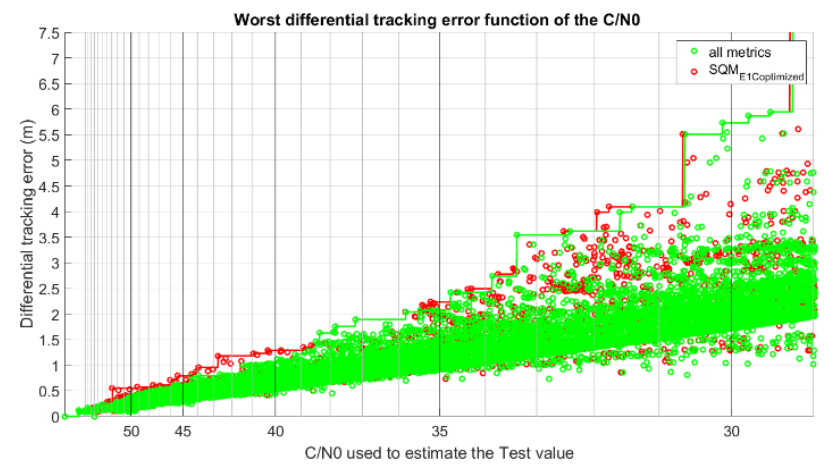

Figure 13. Optimized SQM and 100 metrics $S Q M$ comparison. Galileo E1C.

To satisfy the requirement of 3.5 meters on the maximum differential tracking error, the reference station has to operate with an equivalent $C / N_{0}>33.5 \mathrm{dBHz}$. This $C / N_{0}$ is reached on average on tested operational conditions. 


\section{Galileo E5a}

For Galileo E5a, 21450 distortions are generated (12 TMA, 408 TM-B on area1, 736 TM-B on area2, 5526 TM-C on area1 and $14768 \mathrm{TM}-\mathrm{C}$ on area2). The same concept as with Galileo E1C signal can be applied. In this section the methodology is not detailed as it is done for the Galileo E1C signal.

Regarding the influence of the monitored area, simulations show that a large area has to be monitored. From the 21 monitored correlator outputs chosen in this document to monitor the BPSK(10) autocorrelation function, all correlator outputs must be used to not decrease visibly the efficiency of the SQM.

The distance between two monitored correlator outputs which is equal to $0.1 T_{c}$ cannot be reduced in the Galileo E5a case without degrading performances of SQM.

As with the Galileo E1C signal, difference ratio metrics do not improve the SQM whereas sum ratio metrics do.

Finally an optimized SQM ( $\left.S Q M_{E 5 a_{\text {optimized }}}\right)$ is proposed to monitor Galileo E5a signal distortions defined at the beginning of the document.

$S Q M_{E 5 a_{\text {optimized }}}$ consists of 18 metric $_{x}$ with $x=$ $-1: 0.1:-0.2$ and $x=0.2: 0.1: 1$ in chip unit and 2 metric $_{x+x}$ with $x=0.1: 0.6: 0.7$ in chip unit.

This SQM is not optimal but limits the number of sub-tests to realize, almost reaching performances of a SQM based on 20 metric $_{x}$ plus 10 metric $_{x+x}$ plus 10 metric $_{x-x}$. Figure 14 proposes a performance comparison between $S Q M_{E 5 a_{\text {optimized }}}$ (in red) and the SQM using the 40 metrics (in green).

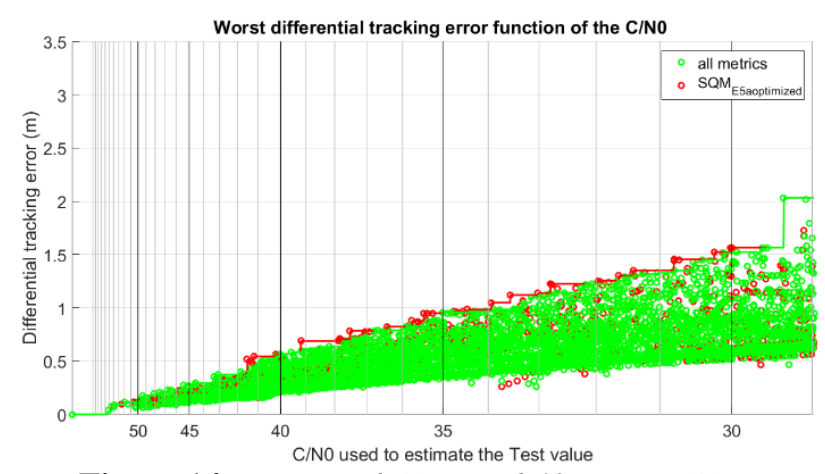

Figure 14. Optimized $S Q M$ and 40 metrics $S Q M$ comparison. Galileo E5a.

For Galileo E5a signal the requirement on the MERR equal to 3.5 meters is satisfied even for the lowest simulated $C / N_{0}$.

\section{CONCLUSION AND FUTURE WORKS}

This paper tackles the design of SQM regarding new GNSS signals: Galileo E5a and Galileo E1C. SQM performances are assessed theoretically for different SQM designs. These performances are dependent upon:

- Distortions from the TM that have to be detected.

- User and reference configurations under discussion.

- $\quad$ Type of metrics used to design the SQM.

In the first part of this document distortions and receiver/reference configurations considered for the study are defined.

In the second part the three metrics used to design different SQMs are introduced: the simple, the difference and the sum ratio metrics. It is assumed that metrics are totally dependent in order to estimate SQM performances in a conservative way. In the meantime some SQM notions are exposed. The main issue in SQM study is the determination of performance thresholds which are directly linked to the metric standard deviations if metric standard deviations are Gaussian. This strong hypothesis was verified in previous works. Nevertheless if this hypothesis is not verified, from performance thresholds estimated in real conditions, SQM performances can still be evaluated from the proposed representation (independently from the $C / N_{0}$ value) presented in this paper using an abacus which gives the theoretical $C / N_{0}$ value associated to thresholds estimated in real conditions. It has been seen from some examples that in the worst case, reference stations are operating with an equivalent theoretical $C / N_{0}=34 \mathrm{dBHz}$ considering unsmoothed metrics and $C / N_{0}=38 \mathrm{dBHz}$ with a $100 \mathrm{sec}$ averaging smoothing on metrics.

In the third part optimized SQMs are proposed for Galileo E1c and Galileo E5a signals. To design these SQMs, 51 monitored correlator outputs for Galileo E1C ( $I_{x}$ with $x=$ -0.25: 0.01: 0.25 $T_{c}$ ) and 21 monitored correlator outputs for Galileo E5a $\left(I_{x}\right.$ with $\left.x=-1: 0.1: 1 T_{c}\right)$ are used. The three metrics introduced in the second part are kept. The optimized SQM consists of 22 metrics for Galileo E1C and in 20 metrics for Galileo E5a.

Some general results are put forward:

- Distortions detection with metrics based on correlator outputs distant from the correlation function main peak ( $>200$ nsec) is more difficult than with metrics based on correlator outputs close to the prompt. Indeed, second order distortions are attenuated according to the damping factor. Moreover, these correlator outputs are more affected by multipath which has the consequence to increase metrics standard deviation.

- The use of additive monitored correlator outputs close to each other $(<10 \mathrm{nsec})$ does not increase detection performance. It is due to the fact that the lowest period of ringing effects considered in this document is equal to $1 /\left(19 \times 10^{-6}\right) \approx 50 \mathrm{nsec}$ and that high frequency phenomena are filtered out by the RF filter applied on the received signal.

- The difference ratio metric is not able to detect symmetric distortions that can have a threatening impact on differential users. 
A more precise selection of metrics used for the SQM could be envisaged. The proposed optimized SQM has to be seen as a compromise between complexity and performance. Fixing the maximum tolerable differential error to 3.5 meters the Galileo E1C optimized SQM is suitable for equivalent theoretical $C / N_{0}>33.5 \mathrm{dBHz}$ whereas the Galileo E5a optimized SQM satisfies the requirement for $C / N_{0}>29 \mathrm{dBHz}$ and lower $C / N_{0}$ values that are not shown. One of the conclusion also valid for the GPS L5 signal is that monitoring a signal BPSK(10) modulated is affordable even with simple SQM design and poor reference receiver conditions. For Galileo E1C a metric smoothing (less than $100 \mathrm{sec}$ should be sufficient) or the use of more complex metrics allows SQM to fulfil required performance of 3.5 meters.

The work performs is realized in a theoretical way. Nonetheless, a method is proposed to adapt theoretical results to reference station conditions. Further works will be to estimate reference station performance thresholds in order to know performances that could be expected in real environment with particular reference receiver filters.

Then, instead of having an optimized SQM, an optimal SQM could be designed following the example of the alpha metric concept [5]. Finally, the approach developed in the paper could be applied to other modulations.

\section{REFERENCES}

[1] R. E. Phelts, "Multicorrelator techniques for robust mitigation of threats to GPS signal quality," Stanford University, California, 2001.

[2] ICAO, "ICAO Convention - Annex 10: Aeronautical Telecommunications - Volume 1: Radio Navigation Aids," 2006.

[3] J.-B. Pagot, P. Thevenon, O. Julien, F. AmarilloFernández, and D. Maillard, "Threat Model Design for new GNSS signals," in Proceedings of the 2016 International Technical Meeting of The Institute of Navigation, Monterey, California, 2016.

[4] M. Irsigler, "Multipath Propagation, Mitigation and Monitoring in the Light of Galileo and the Modernized GPS," Universität der Bundeswehr München, 2008.

[5] R. E. Phelts, T. Walter, and P. Enge, "Toward realtime SQM for WAAS: improved detection techniques," in Proceedings of the 16th International Technical Meeting of the Satellite Division of the Institute of Navigation, 2003, pp. 2739-2749.

[6] R. E. Phelts and T. Walter, "Practical Signal Quality Monitoring for Augmentation Systems," IS GNSS, 2003.

[7] R. E. Phelts, T. Walter, P. Enge, and G. Wong, "Signal Deformation Monitoring for DualFrequency WAAS," in Proceedings of the 2013 International Technical Meeting of The Institute of Navigation, San Diego, California, 2013.
[8] P. Brocard, P. Thevenon, O. Julien, D. Salos, and M. Mabilleau, "Measurement Quality Assessment in Urban Environments Using Correlation Function Distortion Metrics," in Proceedings of the 27th International Technical Meeting of The Satellite Division of the Institute of Navigation, 2014.

[9] J.-M. Sleewaegen and F. Boon, "Mitigating shortdelay multipath: a promising new technique," in Proceedings of ION GPS, 2001, pp. 11-14.

[10] R. E. Phelts, D. M. Akos, and P. Enge, "Robust signal quality monitoring and detection of evil waveforms," in Proceedings of the 13th International Technical Meeting of the Satellite Division of the Institute of Navigation, 2000, pp. 1180-1190.

[11] A. S. Bruce, A. J. Van Dierendonck, A. Jakab, J. Wiebe, and B. Townsend, "Detection of GPS Satellite Signal Failures in Satellite Based Augmentation Systems (SBAS).” Proceedings of the 13th International Technical Meeting of the Satellite Division of The Institute of Navigation, 2000.

[12] R. E. Phelts, E. Altshuler, T. Walter, and P. Enge, "Validating Nominal Bias Error Limits Using 4 years of WAAS Signal Quality Monitoring Data," in Proceedings of the ION 2015 Pacific PNT Meeting, 2015.

[13] P. Thevenon, Q. Tessier, D. Maillard, M. Cabantous, F. Amarillo-Fernández, F. D. O. Salgueiro, and O. Julien, "Detection Performances of Evil Waveform Monitors for the GPS L5 Signal," in 27th International Technical Meeting of The Satellite Division of the Institute of Navigation, 2014.

[14] O. Julien, "Design of Galileo L1F receiver tracking loops," University of Calgary, Canada, 2006.

\section{APPENDIX}

On the appendix the expression of standard deviations applied to metrics presented in the document are derived. Simple ratio, difference ratio and sum ratio metrics standard deviations are estimated in [8] regarding a normalization by the prompt. As discussed, in WAAS reference stations, metrics are based on virtual prompt, it means that metrics can be modelled as:

- The simple ratio metric normalized by a virtual prompt: $\frac{I_{Z}}{I_{X}+I_{Y}}$.

- The difference ratio metric normalized by a virtual prompt: $\frac{I_{Z}-I_{W}}{I_{X}+I_{Y}}$.

- The sum ratio metric normalized by a virtual prompt: $\frac{I_{Z}+I_{W}}{I_{X}+I_{Y}}$.

First of all a model for $I_{x}$ is proposed. This model was already introduced in [9]. The modelling gives a mathematical definition of parameters necessary to compute metrics standard deviation.

Then expressions of theoretical standard deviations are derived. 


\section{Correlator output model}

A simplified model for in phase correlator outputs affected by thermal noise (assuming a constant code and phase delay during the coherent integration time $T_{i n t}$ and assuming that tracking errors are negligible) is given by:

$$
I_{x}=\sqrt{\frac{P}{2}} R_{s}(x)+n_{I}
$$

- $\quad R_{s}$ is the correlation function of the local replica and the filtered received signal.

- $\quad n_{I}$ is the noise on the in-phase component.

- $\quad P$ is the power of the received signal.

Moreover, the noise correlation function at correlator outputs can be modelled using the Wiener Lee relation as it was done in [14]:

$$
\begin{gathered}
R_{n_{I}}(\tau)=\frac{N_{0}}{4 T_{\text {int }}} \int_{-\infty}^{+\infty}\left|H_{R F}(f)\right|^{2 S_{\text {local }}(f) S_{\text {local }}^{*}(f) e^{2 i \pi f \tau} d f} \\
=\frac{N_{0}}{4 T_{\text {int }}} R_{N}(\tau)
\end{gathered}
$$

- $\quad S_{\text {local }}(t)$ is the Fourier transform of the local replica signal.

- $\quad H_{R F}(f)$ is the RF filter transfer function.

- $\quad N_{0}=k_{b} T_{s y s}$ is the noise Gaussian density when the noise is considered white and Gaussian.

- $\quad k_{b}$ is the Boltzmann constant equal to $-228.6 \mathrm{dBW} /$ $\mathrm{K} / \mathrm{Hz}$.

- $\quad T_{\text {sys }}$ is the system noise temperature in degrees on the Kelvin scale.

- $\quad T_{\text {int }}$ is the coherent integration time in sec.

The standard deviation at the correlator outputs is derived from:

$$
\sigma\left(n_{I_{x}}\right)=\sqrt{\frac{P_{n_{I}}}{P_{S}}}=\sqrt{\frac{R_{n_{I}}(0)}{I_{x}{ }^{2}}}=\sqrt{\frac{1}{2 C / N_{0} \times T_{i n t}} \times \frac{R_{N}(0)}{R_{S}^{2}(x)}}
$$

And the covariance can be estimated by:

$$
\begin{aligned}
\operatorname{cov}\left(n_{I_{x}}, n_{I_{y}}\right)= & \frac{R_{n_{I}}(x-y)}{I_{X} I_{Y}} \\
& =\frac{1}{2 C / N_{0} \times T_{\text {int }}} \times \frac{R_{N}(x-y)}{R_{S}(x) R_{S}(y)}
\end{aligned}
$$

Standard deviations and covariance are given for correlator outputs with a mean $\mu_{I_{x}}$ equal to 1 .

Another way to express the standard deviation, covariance and the mean of a correlator output can be obtained by a variable change:

$$
\begin{gathered}
\mu_{I_{x}}=R_{s}(x) \sqrt{2 C / N_{0} T_{\text {int }}} \\
\sigma\left(n_{I_{x}}\right)=\sqrt{R_{N}(0)} \\
\operatorname{cov}\left(n_{I_{x}}, n_{I_{y}}\right)=R_{N}(x-y)
\end{gathered}
$$

Finally, we find the model already proposed in [9] with the difference that the impact of the filter is now taken into account. Indeed, instead of ideal correlation function expression, true correlation functions (which include filtering effect) are adopted in the proposed definition.

\section{Theoretical standard deviations}

The demonstration presented in [8] is applied in this appendix for the simple ratio metric normalized by the virtual prompt. For other metrics, same method could be applied but only results are provided.

Simple ratio metric normalized by a virtual prompt

The first step consists of using the first order development in Taylor series:

$$
\frac{I_{Z}}{I_{X}+I_{Y}} \approx\left(\frac{\mu_{Z}+n_{Z}}{\mu_{X}+\mu_{Y}}\right)\left(1-\frac{n_{X}+n_{Y}}{\mu_{X}+\mu_{Y}}+\left(\frac{n_{X}+n_{Y}}{\mu_{X}+\mu_{Y}}\right)^{2}\right)
$$

The second step consists of the evaluation of $E[\text { metric }]^{2}$. First of all $E[$ metric $]$ is expressed at the second order, zero means and higher order terms are not considered.

$$
\begin{gathered}
E\left[\frac{I_{Z}}{I_{X}+I_{Y}}\right] \approx \frac{1}{\mu_{X}+\mu_{Y}} E\left[\begin{array}{c}
\mu_{Z}-\frac{n_{X} n_{Z}}{\mu_{X}+\mu_{Y}}-\frac{n_{Y} n_{Z}}{\mu_{X}+\mu_{Y}} \\
+\left(n_{X}+n_{Y}\right)^{2} \frac{\mu_{Z}}{\left(\mu_{X}+\mu_{Y}\right)^{2}}
\end{array}\right] \\
=\frac{1}{\mu_{X}+\mu_{Y}}\left(\begin{array}{c}
\mu_{Z}-\frac{E\left[n_{X} n_{Z}\right]}{\mu_{X}+\mu_{Y}}-\frac{E\left[n_{Y} n_{Z}\right]}{\mu_{X}+\mu_{Y}} \\
+\left(E\left[n_{X}^{2}\right]+E\left[n_{Y}^{2}\right]+2 E\left[n_{X} n_{Y}\right]\right) \frac{\mu_{Z}}{\left(\mu_{X}+\mu_{Y}\right)^{2}}
\end{array}\right)
\end{gathered}
$$

The square of the last expression is then written:

$$
\begin{gathered}
E\left[\frac{I_{Z}}{I_{X}+I_{Y}}\right]^{2} \approx \frac{1}{\left(\mu_{X}+\mu_{Y}\right)^{2}} \\
\left(\begin{array}{c}
\mu_{Z}^{2}-2 \frac{\mu_{Z}}{\mu_{X}+\mu_{Y}}\left(E\left[n_{X} n_{Z}\right]+E\left[n_{Y} n_{Z}\right]\right) \\
+2\left(E\left[n_{X}^{2}\right]+E\left[n_{Y}^{2}\right]+2 E\left[n_{X} n_{Y}\right]\right) \frac{\mu_{Z}^{2}}{\left(\mu_{X}+\mu_{Y}\right)^{2}}
\end{array}\right)
\end{gathered}
$$

The third step consists of the estimation of $E\left[\right.$ metric $\left.^{2}\right]$. The expression is also given at the second order (zero means and higher order terms are not considered):

$$
\begin{gathered}
E\left[\left(\frac{I_{Z}}{I_{X}+I_{Y}}\right)^{2}\right] \approx \\
E\left[\left(\frac{\mu_{Z}+n_{Z}}{\mu_{X}+\mu_{Y}}\right)^{2}\left(1-\frac{n_{X}+n_{Y}}{\mu_{X}+\mu_{Y}}+\left(\frac{n_{X}+n_{Y}}{\mu_{X}+\mu_{Y}}\right)^{2}\right)^{2}\right] \\
=\frac{1}{\left(\mu_{X}+\mu_{Y}\right)^{2}} E\left[\begin{array}{c}
\mu_{Z}^{2}+n_{Z}^{2}-4 \frac{\mu_{Z}}{\left(\mu_{X}+\mu_{Y}\right)} n_{Z}\left(n_{X}+n_{Y}\right) \\
+3 \frac{\mu_{Z}^{2}}{\left(\mu_{X}+\mu_{Y}\right)^{2}}\left(n_{X}+n_{Y}\right)^{2}
\end{array}\right]
\end{gathered}
$$

The last step consists of the estimation of the metric standard deviation using its definition:

$$
\sigma^{2}(\text { metric })=E\left[\text { metric }^{2}\right]-E[\text { metric }]^{2}
$$




$$
\left[\begin{array}{c}
\sigma^{2}\left(\frac{I_{Z}}{I_{X}+I_{Y}}\right)=\frac{1}{\left(\mu_{X}+\mu_{Y}\right)^{2}} \\
-4 \frac{\mu_{Z}}{\left(\mu_{X}+\mu_{Y}\right)}\left(E\left[n_{X} n_{Z}\right]+E\left[n_{Y} n_{Z}\right]\right) \\
+3 \frac{\mu_{Z}^{2}}{\left(\mu_{X}+\mu_{Y}\right)^{2}}\left(E\left[n_{X}^{2}\right]+E\left[n_{Y}^{2}\right]+2 E\left[n_{X} n_{Y}\right]\right) \\
-\left(\begin{array}{c}
\mu_{Z}^{2}-2 \frac{\mu_{Z}}{\mu_{X}+\mu_{Y}}\left(E\left[n_{X} n_{Z}\right]+E\left[n_{Y} n_{Z}\right]\right) \\
\left.+2\left(E\left[n_{X}^{2}\right]+E\left[n_{Y}^{2}\right]+2 E\left[n_{X} n_{Y}\right]\right) \frac{\mu_{Z}^{2}}{\left(\mu_{X}+\mu_{Y}\right)^{2}}\right)
\end{array}\right] \\
\frac{1}{\left(\mu_{X}+\mu_{Y}\right)^{2}}\left[\mu_{Z}^{2}+E\left[n_{Z}^{2}\right]\right. \\
-2 \frac{\mu_{Z}}{\left(\mu_{X}+\mu_{Y}\right)}\left(E\left[n_{X} n_{Z}\right]+E\left[n_{Y} n_{Z}\right]\right) \\
+\frac{\mu_{Z}^{2}}{\left(\mu_{X}+\mu_{Y}\right)^{2}}\left(E\left[n_{X}^{2}\right]+E\left[n_{Y}^{2}\right]\right. \\
\left.\left.+2 E\left[n_{X} n_{Y}\right]\right)\right]
\end{array}\right.
$$

Finally the standard deviation of a simple ratio metric normalized by a virtual prompt can be modelled as:

$$
\begin{aligned}
& \sigma\left(\frac{I_{Z}}{I_{X}+I_{Y}}\right) \\
& =\sqrt{\frac{\mu_{Z}^{2}}{\left(\mu_{X}+\mu_{Y}\right)^{2}}\left[\begin{array}{c}
\frac{\sigma^{2}\left(n_{Z}\right)}{\mu_{Z}^{2}} \\
+\frac{\sigma^{2}\left(n_{X}\right)+\sigma^{2}\left(n_{Y}\right)+2 \operatorname{cov}\left(n_{Y} n_{X}\right)}{\left(\mu_{X}+\mu_{Y}\right)^{2}} \\
-2 \frac{\operatorname{cov}\left(n_{X} n_{Z}\right)+\operatorname{cov}\left(n_{Y} n_{Z}\right)}{\mu_{Z}\left(\mu_{X}+\mu_{Y}\right)}
\end{array}\right]}
\end{aligned}
$$

Difference ratio metric normalized by a virtual prompt Using the same process, the metric standard deviation of a difference ratio metric can be modelled as:

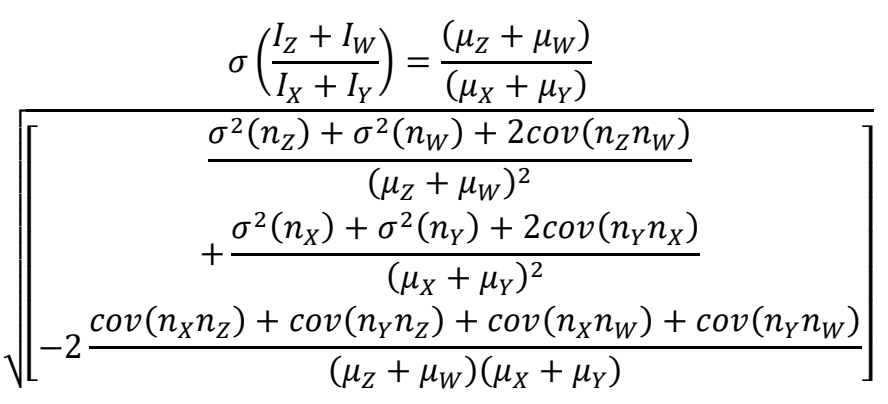

Sum ratio metric normalized by a virtual prompt Using the same process, the metric standard deviation of a sum ratio metric can be modelled as:

$$
\sigma\left(\frac{I_{Z}-I_{W}}{I_{X}+I_{Y}}\right)=\frac{\left(\mu_{Z}+\mu_{W}\right)}{\left(\mu_{X}+\mu_{Y}\right)}
$$

$$
\sqrt{\left[\begin{array}{c}
\frac{\sigma^{2}\left(n_{Z}\right)+\sigma^{2}\left(n_{W}\right)-2 \operatorname{cov}\left(n_{Z} n_{W}\right)}{\left(\mu_{Z}+\mu_{W}\right)^{2}}+ \\
\frac{\sigma^{2}\left(n_{X}\right)+\sigma^{2}\left(n_{Y}\right)+2 \operatorname{cov}\left(n_{Y} n_{X}\right)}{\left(\mu_{X}+\mu_{Y}\right)^{2}}
\end{array}\right]}
$$

\section{Abacuses for Galileo E1C and E5a signals}

For each signal, an abacus is necessary to make the link between metrics standard deviation and theoretical $C / N_{0}$. The abacus is also dependent on the metric normalization. Considering simple ratio metrics (normalized by a virtual prompt) introduced in the publication, the abacus for Galileo E1C is shown on the top and the abacus for Galileo E5a is shown on the bottom.
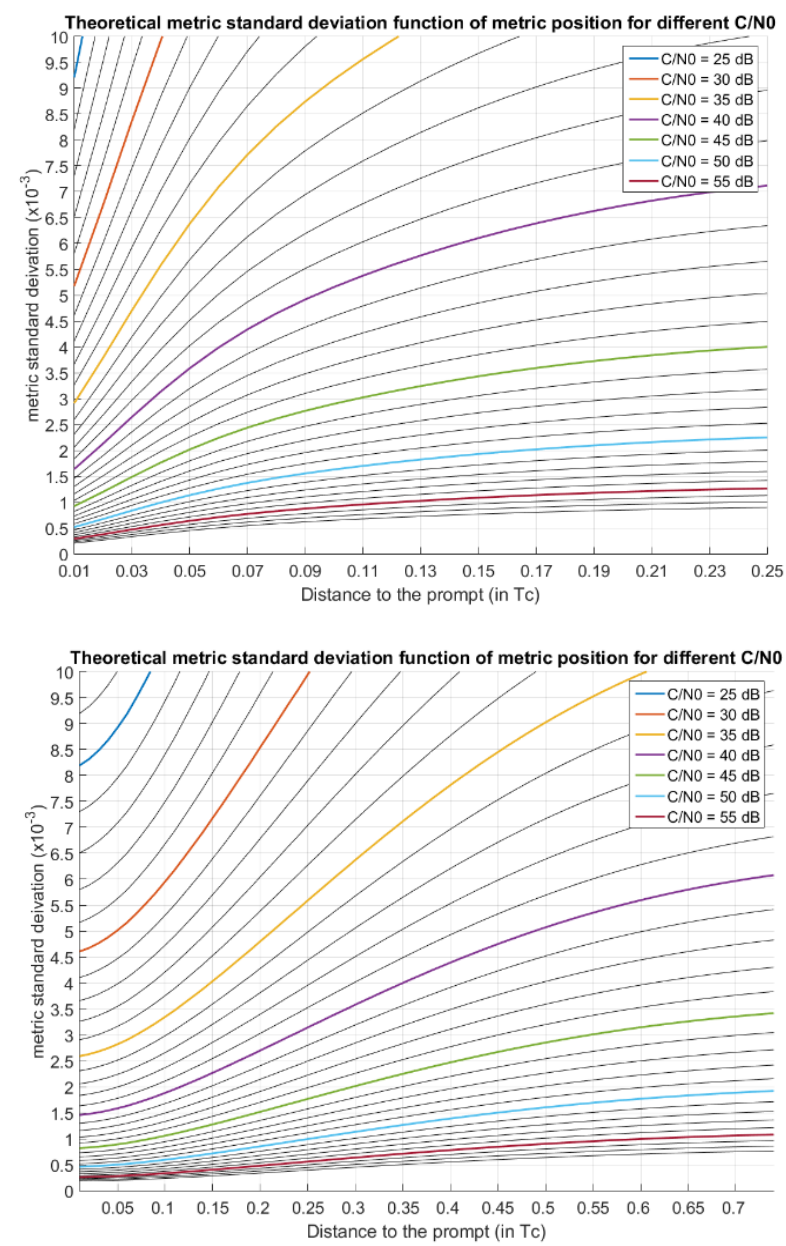

Figure A-1. Metric standard deviations compared to theoretical values. Galileo E1C signal on the top, Galileo E5a signal on the bottom. 